\title{
The Confronting COPD International Survey: patients hardly know they have COPD
}

\author{
P.N.R. Dekhuijzen
}

An important part of the daily clinical practice of the pulmonologist is related to patients with chronic obstructive pulmonary disease (COPD). Management of stable COPD includes recommendations regarding lifestyle and physical exercise, and optimal individually tailored pharmacotherapy will be considered satisfactory by the physician if lung function decline is limited and frequent and/or severe exacerbations are prevented. Many international and national guidelines have been developed, which include practical issues regarding management, monitoring etc. $[1,2]$.

Research in COPD has expanded rapidly in recent years. Its main aims have been to unravel the pathobiology, to explore similarities and differences compared to asthma, to find noninvasive biomarkers that indicate the presence and severity of inflammation and oxidative stress, and to analyse the short- and longterm efficacy of therapeutic interventions.

Relatively little is known about the patient's own perception of their health status with regard to COPD. Obviously, it is important to explore this significant issue because there may be a discrepancy between the doctor's perception of the impact of COPD in the patient and the patient's own perception of their health status. A clear example of such a discrepancy was recently shown in the Asthma Insights and Reality in Europe (AIRE) study [3], showing that the impact of asthma was much larger from the patient's perspective than many doctors would have anticipated.

In the present issue of the European Respiratory Journal, RENNARD et al. [4] show real-life data collected from subjects with a known or suspected diagnosis of COPD. One of the interesting conclusions from this "Confronting COPD International Survey" is that subjects with COPD underestimate their morbidity, as evidenced by a high proportion of subjects with limitations in daily life activities, a marked loss of work, and frequent use of health services. A remarkable finding was that patients tend to underestimate the severity of the disease, as evidenced by the discrepancy between breathlessness score and their own estimation of severity. The prevalence of COPD in the population of smokers $\geqslant 45$ yrs of age appeared

Correspondence: P.N.R. Dekhuijzen, Dept of Pulmonology, University Medical Centre Nijmegen, PO Box 9101, 6500 HB Nijmegen, the Netherlands. Fax: 31 243610324. E-mail: r.dekhuijzen@long. azn.nl to be $\sim 4 \%$, with relatively little variation among the countries surveyed.

How should these data be interpreted and put into perspective? The data reported on limitations in daily life are impressive, but, as a consequence of the design of the survey, they are not accompanied by lung function data. About $80 \%$ of the studied population underwent some kind of lung function testing in the previous year, but only $55 \%$ underwent any tests other than peak flow registration. In view of the significant limitations in daily life activities reported in the present study, the disease state may have been underdiagnosed and/or the severity underestimated.

As would be expected, only $\sim 55 \%$ of the subjects reported use of inhaled bronchodilators. This strongly suggests underestimation of the severity of the disease state and undertreatment in these subjects, despite significant complaints and limitations. The extent of this misclassification and undertreatment cannot be assessed in the present study design, but might be quite large.

Another issue is the apparent discrepancy between the intensity of breathlessness and the patient's own assessment of severity of disease. Why do patients with severe breathlessness judge their disease as mild to moderate? Is this because they accept their breathlessness as a part of getting older? Do they think that "chronic bronchitis" is not a severe disease? Are they reluctant to call their disease severe because they feel that smoking-related COPD is self-inflicted? Or do they compare their disease state with their "agematched peers" with cardiovascular disease or cancer?

There are some important lessons that can be learnt from these data. First, patients need to be made aware of the severity of the disease. This could be achieved by explaining to patients that they have COPD (if confirmed by appropriate lung function tests) and by giving a detailed description of their abnormalities (i.e. emphysema or "chronic obstructive bronchitis" or "chronic obstructive bronchiolitis" or combinations). Secondly, correct labelling of the disease appears to be important, as in the present survey only $20 \%$ of the subjects reported to have COPD, indicating that correct and unanimous labelling of these patients need improving. Ensuring common use of this term would help to increase public awareness of this severe and disabling disorder within and among countries. Thirdly, awareness among physicians in primary and secondary care should also be intensified. This is likely to reinforce both secondary prevention in terms of 
smoking cessation, adjustments in lifestyle etc., but hopefully also primary prevention of this insidiously starting disease.

\section{References}

1. Siafakas NM, Vermeire P, Pride NB, et al. Optimal assessment and management of chronic obstructive pulmonary disease (COPD). The European Respiratory Society Task Force. Eur Respir J 1995; 8: 1398-1420.

2. Pauwels RA, Buist AS, Calverley PMA, Jenkins CR, Hurd SS, on behalf of the GOLD Scientific Committee.
Global strategy for the diagnosis, management, and prevention of chronic obstructive pulmonary disease: NHLBI Global Initiative for Chronic Obstructive Lung Disease (GOLD) Workshop Summary. Am J Respir Crit Care Med 2001; 163: 1256-1276.

3. Rabe KF, Vermeire PA, Soriano JB, Maier WC. Clinical management of asthma in 1999: the Asthma Insights and Reality in Europe (AIRE) study. Eur Respir J 2000; 16: 802-807.

4. Rennard S, Decramer M, Calverley PMA, et al. Impact of COPD in North America and Europe in 2000: subjects' perspective of Confronting COPD International Survey. Eur Respir J 2002; 20: 799-805. 